\title{
A case series on current management options of tubal ectopic pregnancy in a tertiary care unit
}

\author{
G H K K Gunawardena ${ }^{1}$, K G W Priyananda ${ }^{2}$, G K C Jayalath ${ }^{3}$, D M P Disanayake ${ }^{4}$
}

Sri Lanka Journal of Obstetrics and Gynaecology 2009; 31: 16-19

\begin{abstract}
Objective: To evaluate the feasibility of different management options of ectopic pregnancy in a local setup.
\end{abstract}

Methods: This was a descriptive cross sectional study carried out at the Teaching Hospital, Peradeniya, Sri Lanka. All the patients who were diagnosed to have ectopic pregnancy during the study period of one year (2008) were included.

The data collection was completed at the time of discharge of each patient. Information included, age, parity, period of gestation, clinical presentation, risk factors for ectopics, diagnosis (transvaginal scan and beta hCG) and type of treatment options (open surgery, laparoscopy, medical management and expectant management). A descriptive analysis of data was done in 21 patients with ectopic pregnancies.

Results: Twenty one ectopic pregnancies were managed during the study period. A majority of cases (clinically stable patients with small gestational sac) $76 \%(16 / 21)$ were managed using treatment options, with continuous monitoring of each patient other than open surgery. Four clinically unstable patients and one patient with a large ectopic sac were subjected to laparotomy, while 8 clinically stable patients were offered laparoscopy, 7 systemic administrations of methotrexate and expectant management.

Conclusions: It was found that most of the cases could be managed by treatment options other than open surgery. Early detection, clinical stability and size of the ectopic sac were

\footnotetext{
${ }^{1}$ Consultant Obstetrician and Gynaecologist,

${ }^{2}$ Senior Registrar in Obstetrics and Gynaecology,

${ }^{3}$ Senior Registrar in Obstetrics and Gynaecology,

${ }^{4}$ Senior House Officer, Teaching Hospital, Peradeniya.

Corresponding author: G H K K Gunawardena

E-mail:samodhar@yahoo.com
}

important determiners in the success of these new treatment options. Analysis of results shows there is no difference between each management modalities.

Key Words: Tubal ectopic pregnancy, laparoscopic surgery in ectopics, medical treatment of ectopics, expectant management in ectopics.

Abbreviations: EP - Ectopic pregnancy, MTX Methotrexate, TVS - Transvaginal ultrasound scan, hCG - Human choronic gonadotrophins.

\section{Introduction}

Ectopic pregnancy (EP) occurs in 1\% of pregnant women, and may seriously compromise women's health and future fertility ${ }^{1}$. Currently, EPs can often be diagnosed before deterioration of the patient's condition and it has changed the former clinical picture of a life-threatening disease into a more benign condition in frequently asymptomatic patients.

The cornerstone in the diagnosis is the use of noninvasive diagnostic algorithms integrating transvaginal ultrasound and serum beta hCG measurement. The use of diagnostic laparoscopy has been the gold standard of diagnosis ${ }^{1,2}$. All types of investigations need to be integrated with the clinical picture of the patient to reach a more probable diagnosis of the condition.

Timely diagnosis allows the clinician to consider the full range of treatment options. This is important since the focus of contemporary therapeutic management exceeds the narrow aim of immediate treatment success (i.e. elimination of the EP) and includes retaining optimal fertility for those women desiring future pregnancy ${ }^{3}$.

To date, therapeutic options for women with tubal EP are surgical, medical treatment or expectant management. In surgery, laparoscopy is now the accepted approach to perform salpingostomy, salpingectomy or milking of the tube and aspiration. Concerning medical treatment, systemic administration of methotrexate (MTX), has gained acceptance 
in selected patients. It is given intramuscularly (IM), either in a fixed multiple dose regimen alternated with folinic acid or in a single-dose regimen without folinic acid. Expectant management has been advocated based on the knowledge that the natural course of many early EPs is a self-limiting process and ultimately results in tubal abortion or re-absorption ${ }^{3,4}$.

A well recognized hazard after salpingostomy, systemic MTX treatment and expectant management is persistent of trophoblastic cells. Persistent trophoblast may lead to the recurrence of clinical symptoms and is an indication for additional treatment ${ }^{4}$. Serum hCG monitoring enables the timely detection of inadequately declining serum hCG concentrations after treatment ${ }^{5}$.

This study aims to describe different management options of ectopic pregnancies in a tertiary care center.

\section{Material and methods}

Prospective analysis of data from ectopic pregnancies which were managed in the ward 18, the Teaching Hospital Peradeniya during the year 2008. The definitive or probable diagnosis of ectopic pregnancy was reached by using patient's clinical picture, serum beta hCG levels, transvaginal ultrasound scan (TVS) and/or laparoscopy.

Data collection was done of all patients who were diagnosed to have ectopic pregnancies during the study period. They were categorized according to the type of management option that they have undergone, such as open surgery (salpingectomy or salpingostomy), laparoscopic surgery (salpingectomy or salpingostomy), medical management (methotrexate) and expectant management.

The mode of treatment was decided by the overall assessment of the patient's clinical stability, sonographic findings (size of the ectopic sac, presence or absence of fetal heart activity and haemoperitoneum), serum beta hCG levels, and 2 beta hCG samples (48hrs apart) that were done on occasions where ultrasound scans were inconclusive. Patient's fertility wishes and patient's personal preference after counseling.

\section{Criteria for methotrexate therapy ${ }^{6}$}

a) Absolute indications

1. Haemodynamically stable without active bleeding or signs of hemoperitoneum

2. Patient desires future fertility

3. Non laparoscopic diagnosis

4. Patient able to return for follow-up care
5. General anesthesia poses risk

6. Patients has no contraindications to methotrexate

b) Relative indications

1. Unruptured mass $<3.5 \mathrm{~cm}$ at greatest dimension

2. No fetal cardiac activity

3. Beta hCG limit does not exceed a predetermined value $(>10,000 \mathrm{mil} / \mathrm{l})$

\section{Exclusion criteria of methotrexate therapy ${ }^{6}$}

a) Absolute

1. Breastfeeding

2. Immunodeficiency

3. Abnormal creatinine $(>1.3 \mathrm{mg} / \mathrm{dl})$ and chronic renal disease.

4. Liver disease

5. Pre-existing blood dyscrasiasias

6. Peptic ulcer disease

7. Active pulmonary disease

8. Known sensitivity to methotrexate

b) Relative

1. Gestational sac $>3.5 \mathrm{~cm}$

2. Cardiac activity

Intense clinical monitoring and re-estimation of serum beta hCG levels have been used to assess the success of the treatment method.

A descriptive analysis of data was done in each category of treatment options as relevant to parity, gestation, clinical presentation, risk factors, future fertility wishes, size of ectopic sac and serum beta hCG.

\section{Results}

The mean age was 28.3 years in open surgery group and it was 28.5 years in women who were categorized as new method of treatment. The mean POA was 8 weeks and 3 days for open surgery and 7 week and 6 days in other group.

21 cases of ectopic pregnancies were managed during the study period. Majority of cases 76\% (16/21) were managed by treatment options other than the traditional method of laparotomy which is associated with much post-operative morbidity.

Four clinically unstable patients suggestive of having ruptured ectopic pregnancies were managed using open surgery or alternatively salpingectomy or salpingostomy. One clinically stable patient had to undergo laparotomy due to the large size of ectopic sac $(36 \mathrm{~mm})$ and the presence of fetal heart activity. 
Eight patients underwent laparoscopic surgeries or alternatively salpingectomy or salpingostomy. All patients were clinically stable and majority had absent or a minimal amount of free fluid suggestive of unruptured ectopic sacs or leaking ectopics. Six cases presented with clinical symptoms while 2 cases were detected by routing pregnancy ultrasound scans.

During the study period 7 ectopic pregnancies were managed medically by methotrexate administration. Methotrexate therapy was successful in 6 cases while in one case laparoscopy was used. This was due to the rising level of beta hCG in spite of multiple dose therapy. Single dose therapy was successful in 5 out of 7 cases with gradual decline of serum beta hCG. Two cases were treated with multiple doses ( 2 doses) as they had rising beta hCG levels in excess of $10000 \mathrm{miu}$.

One patient was offered expectant management after proper counseling and patient presented with clinical symptoms but no ultrasound evidence of intrauterine pregnancy or ectopic gestation even though her serum beta hCG suggestive of a pregnancy. Subsequently she had gradual decline of beta hCG which may suggestive of resolving ectopic pregnancy.

For statistical analysis we classified open surgery as traditional method and all others (medical management, laparoscopy and expectant management) as new methods.

\section{Discussion}

Early diagnosis of ectopic is essential when deciding several treatment options. Analysis of 5 cases of laparotomy suggests late presentation of the condition with clinical instability was led to open surgery. Therefore it is vital to make an early diagnosis because haemodynamic stability is the most important criterion for selection of the patient for other treatment options $^{7}$.

Early diagnosis has altered the clinical presentation of ectopic pregnancy from that of a life threatening surgical emergency to a more benign situation. Our findings show early diagnosis of majority of cases $(76 \%)$, help us to offer several other treatment options for ectopic pregnancies.

Laparoscopy carries more advantages than laprotomy in clinically stable patients as it reduces much of post-operative morbidity. Additionally, it can be used as a definitive diagnostic tool in cases of diagnostic dilemma ${ }^{3}$. However, a combination of the clinical features, quantitative beta-hCG and highresolution transvaginal ultrasound scan has enabled an early and accurate diagnosis of ectopic pregnancy to be made without resorting to laparoscopy ${ }^{8}$.

Considering the 7 cases of methotrexate therapy, clinical stability of the patient is the key to success. In addition to that absence of fetal heart activity, absence of haemoperitoneum and serum beta hCG level below $10000 \mathrm{miu} / \mathrm{ml}$ have shown better outcome. Rising level of beta hCG and presence of fetal heart activity have shown high failure rate even with multiple doses ${ }^{4,7}$. One trial has shown the presence of cardiac activity has higher failure rate than absence of cardiac activity $(14.3 \% \text { vs } 4.4 \%)^{4}$. The presence of fetal cardiac activity is not an absolute contraindication for methotrexate therapy. Therefore even those patients could be treated by methotrexate provided with a careful monitoring system ${ }^{7}$.

Expectant management may be an option for the clinically stable patient with a small ectopic pregnancy or pregnancy of unknown origin and low or declining beta-hCG values, who is willing and able to comply with close follow up. Overall, if the initial serum concentration of beta hCG is less than $1000 \mathrm{miu} / \mathrm{ml}$, expectant management is successful in up to $88 \%$ of patients ${ }^{12}$. However in our case series we managed only one patient expectantly.

\section{Conclusions}

Management of tubal ectopics has been changed over the past few years with the introduction of new treatment modalities (from open surgery to laparoscopic surgery, medical and expectant management). Early detection is the key to the success of new treatment options with newer diagnostic tools (serum beta-hCG and high resolution TVS). With our experience of management of ectopic pregnancy, we found most of the cases could be managed by laparoscopic surgeries and systemic administration of methotrexate safely. However proper patient selection before treatment and continuous monitoring of progress is vital to gain a better outcome.

\section{References}

1. Condous G, Okaro E, Khalid A, Timmerman DL, Zhou Y, van HS, Bourne T. The use of a new logistic regression model for prediciting the outcome of pregnancies of unknown location. Hum Reprod 2004; 19: 1900-10.

2. Ankum WM, Van der Veen F, Hamerlynck HV, Lammes FB. Suspected ectopic pregnancy. J Reprod Med 1995; 40: $525-8$.

Sri Lanka Journal of Obstetrics and Gynaecology 
3. Bouyer J, Job-Spira N, Pouly J, Coste J, Germain E, Fernandez $\mathrm{H}$. Fertility following radical, conservativesurgical or medical treatment for tubal pregnancy: a population-based study. BJOG 2000; 107: 714-21.

4. Elmoghazy DAM, Nour-El-Dine NM. Prevention of persistent ectopic pregnancy with single dose methotrexate after surgical conservation of the tube. In: Abstracts of the XVI FIGO World Congress of Obstetrics and Gynecology 2000: 57.

5. Vermesh M, Silva PD, Rosen GF, Stein AL, Fossum GT, Sauer MV. Management of unruptured ectopic gestation by linear salpingostomy: a prospective, randomized clinical trial of laparoscopy versus laparotomy. Obstet Gynecol 1989; 73: 400-4.

6. Liberato VM, Stephamie BT, current management of ectopic pregnancy. Obstet Gynecol Clin N Am 2007; 34: 403-19.

7. Ashis S, Diana HF, non surgical diagnosis and management of ectopic pregnancy. The Obstetrician and Gynaecologist 2003; 5: 29-33.

8. Ling FW. Stovall TG. Update on the diagnosis and management of ectopic pregnancy. In:Rock. JA. editor. advances in obstetrics and gynaecology: Chicago: Mosby: 1994: 55-83. 